\title{
Associations between apolipoprotein E genotypes and serum levels of glucose, cholesterol, and triglycerides in a cognitively normal aging Han Chinese population
}

This article was published in the following Dove Press journal:

Clinical Interventions in Aging

9 July 2014

Number of times this article has been viewed

\author{
Qing-Qing Tao',* \\ Yan Chen ${ }^{2,3, *}$ \\ Zhi-Jun Liu' \\ Yi-Min Sun' \\ Ping Yang' \\ Shen-Ji Lu' \\ Miao $\mathrm{Xu}^{\prime}$ \\ Qin-Yun Dong' \\ Jia-Jun Yang ${ }^{2}$ \\ Zhi-Ying Wu' \\ 'Department of Neurology and \\ Institute of Neurology, Huashan \\ Hospital, Shanghai Medical College, \\ Fudan University, ${ }^{2}$ Department of \\ Neurology, Sixth People's Hospital \\ Affiliated to Shanghai Jiao Tong \\ University, ${ }^{3}$ Department of Medicine, \\ Shanghai Fengxian District Central \\ Hospital, Shanghai, People's Republic \\ of China
}

*These authors contributed equally to this work

Correspondence: Zhi-Ying Wu

Department of Neurology and Institute

of Neurology, Huashan Hospital, Shanghai

Medical College, Fudan University,

12 Wulumuqi Zhong Road, Shanghai

200040, People's Republic of China

Tel +86 2l 6248342 I

Fax +86 2I 6248 342I

Email zhiyingwu@fudan.edu.cn

Jia-Jun Yang

Department of Neurology, Sixth People's Hospital Affiliated to Shanghai Jiao Tong University, 600 Yishan Road, Shanghai

200233, People's Republic of China

Tel +862138297738

Fax +86 2I 38297738

Email yangjiajunfzy@sina.com
Purpose: To determine the associations between apolipoprotein E ( $A P O E$ ) genotypes and serum levels of glucose, total cholesterol, and triglycerides in a cognitively normal aging Han Chinese population.

Methods: There were 1,003 cognitively normal aging subjects included in this study. APOE genotypes were analyzed and biochemical parameters were tested. All the subjects were divided into three groups according to $A P O E$ genotypes: (1) E2/2 or E2/3 (APOE E2); (2) E3/3 (APOE $\mathrm{E} 3$ ); and (3) E2/4, E3/4, or E4/4 ( $A P O E \mathrm{E} 4)$. Correlations of serum levels of glucose, total cholesterol, and triglycerides with $A P O E$ genotypes were assessed.

Results: E2, E3, and E4 allele frequencies were found to be $6.2 \%, 82.1 \%$, and $11.7 \%$, respectively. Serum levels of total cholesterol were higher in the APOE E4 group $(P<0.05)$. A higher level of total cholesterol was associated with the E4 allele (adjusted odds ratio 1.689, 95\% confidence interval 1.223-2.334, $P<0.01$ ). However, no association was found between APOE status and serum levels of glucose (adjusted odds ratio $0.981,95 \%$ confidence interval $0.720-1.336, P=0.903$ ) or total triglycerides (adjusted odds ratio $1.042,95 \%$ confidence interval $0.759-1.429, P=0.800)$.

Conclusion: A higher serum level of total cholesterol was significantly correlated with $A P O E$ E4 status in a cognitively normal, nondiabetic aging population. However, there was no correlation between $A P O E$ genotypes and serum levels of glucose or total triglycerides.

Keywords: Alzheimer's disease, $A P O E$, glucose level, cholesterol, triglycerides

\section{Introduction}

Apolipoprotein E (APOE), a major cholesterol carrier in the brain, has been proven to be associated with the pathology of Alzheimer's disease (AD). APOE has three common alleles including E2, E3, and E4, encoding three protein isoforms. The E4 allele of $A P O E$ was consistently recognized as a risk factor for AD. ${ }^{1}$

APOE can alter cholesterol homeostasis, lipid transport, $\mathrm{A} \beta$ deposition, and clearance in an isoform-dependent manner. ${ }^{2-4}$ During the last few decades, studies performed in different populations have explored the associations between $A P O E$ genotypes and serum lipid metabolism; the studies have reached an agreement that the $A P O E$ E4 carrier is correlated with a higher serum of total cholesterol (TC) level as well as a low-density lipoprotein cholesterol level. ${ }^{5,6}$

In addition to its well-recognized roles mentioned above, recent studies have suggested that $A P O E$ might also be involved in glucose metabolism. ${ }^{7}$ Diabetes and perturbed glucose metabolism have been proven as risk factors for 
cognitive impairment. ${ }^{8}$ Dysregulation of cerebral glucose metabolism was also observed in both mild cognitive impairment and AD patients. ${ }^{9}{ }^{10}$ Recently, one study reported that higher glucose levels may be a risk factor for dementia, ${ }^{11}$ and poor control of peripheral glucose levels resulted in worse cognitive function and accelerated the decline. ${ }^{12,13}$ Another study reported that higher serum glucose levels in cognitively normal, nondiabetic adults were associated with cerebral hypometabolism in vulnerable brain areas of $\mathrm{AD}$ patients and suggested that these effects might be independent of $A P O E$ E4 status. ${ }^{14}$ However, so far the correlations between $A P O E$ genotypes and serum glucose level have not been clearly identified.

Here, 1,003 cognitively normal, nondiabetic Han Chinese aging subjects were selected from a 2,149 aging population recruited from community epidemiologic investigations to explore the associations of $A P O E$ genotypes with the levels of serum glucose as well as TC and total triglycerides (TG). To the best of the authors' knowledge, this is the first study on the associations between $A P O E$ genotypes with serum levels of glucose, TC, and TG in a cognitively normal Han Chinese aging population from mainland People's Republic of China.

\section{Methods and materials Subjects}

This study involved 1,003 cognitively normal aging subjects selected from a 2,149 aging population recruited from community epidemiologic investigations during 2011-2012. Written consents were obtained from subjects or their legally authorized caregivers. An interview about age, sex, year of education, medical history, and history of smoking and drinking was performed. Body mass index was calculated as weight divided by height squared $\left(\mathrm{kg} / \mathrm{m}^{2}\right)$. Mini-Mental State Examination and complete physical and neurologic examinations were also evaluated. As most of the subjects were illiterate, Mini-Mental State Examination score $>18$ was used as an inclusion criterion. ${ }^{15,16}$ All of the selected subjects denied memory or any other cognitive impairment. Additional exclusion criteria were diabetes mellitus, hepatic disease, hypothyroidism, and history of receiving drugs that affect serum glucose and lipids. Blood collections were performed according to the standard process. Briefly, the subject was positioned in a chair, placing the tourniquet 4 inches above the selected puncture site on the subject. A vein was selected and the area cleansed. The needle was then inserted through the skin into the lumen of the vein. Ethylenediaminetetraacetic acid-containing tubes were used for blood sample collection for the isolation of DNA; clot activating gel-containing serum separator tubes were used for blood sample collection for assessment of the biochemical parameters. This study was approved by the ethics committee of Huashan Hospital (Shanghai, People's Republic of China).

\section{Genotyping of $A P O E$}

For $A P O E$ genotype determinations, genomic DNA was extracted from peripheral blood using a Blood Genomic DNA Extraction Kit (Qiagen NV, Venlo, the Netherlands). The APOE genotypes were determined by multiplex amplification refractory mutation system polymerase chain reaction according to the method previously described. ${ }^{17}$

\section{Measurement and analysis serum levels of glucose, TC, and TG}

Fasting serum glucose levels were assessed by hexokinase method on an autoanalyzer (Dimension ${ }^{\circledR}$ Xpand Plus; Siemens AG, Erlangen, Germany). Serum TC and TG levels were determined on the same autoanalyzer (Dimension Xpand Plus). According to the People's Republic of China's clinical laboratory criteria for lipid values, serum TC level $>5.7 \mathrm{mmol} / \mathrm{L}$, and TG level $>1.8 \mathrm{mmol} / \mathrm{L}$ was defined as hypercholesterolemia and hypertriglyceridemia. For glucose, fasting venous blood glucose level $>6.1 \mathrm{mmol} / \mathrm{L}$ was defined as hyperglycemia.

\section{Statistical analysis}

One-way analysis of variance was performed to assess the differences in the mean value of glucose, TC, and TG in the different groups. Categorical data such as gender, history of smoking, and history of alcohol drinking were evaluated by chi-square tests. Possible associations between $A P O E$ genotype and serum levels of glucose, TC, and TG were determined by logistic regression analyses. Serum levels of glucose, TG, and TC were used as dependent variables which were dichotomized according to the People's Republic of China's clinical laboratory criteria for hypercholesterolemia, hypertriglyceridemia, and hyperglycemia. Age, sex, body mass index, and history of smoking and alcohol drinking were used as covariates. All statistical analyses were performed using SPSS version 13.0 (SPSS Inc., Chicago, IL). $P<0.05$ was considered statistically significant.

\section{Results}

\section{Characteristics of subjects} with different $A P O E$ genotypes

The results of allele and genotype frequencies are shown in Table 1. The frequency of $A P O E$ E3 was greatest (82.1\%), 
Table I Allele frequencies and prevalence of APOE genotypes in a cognitively normal aging Han Chinese population

\begin{tabular}{llll}
\hline APOE & Male $(\mathbf{n = 4 3} \mathbf{I})$ & Female $(\mathbf{n = 5 7 2})$ & Combined $(\mathbf{n}=\mathbf{I}, \mathbf{0 0 3})$ \\
\hline E2 & $46(5.3 \%)$ & $79(6.9 \%)$ & $125(6.2 \%)$ \\
E3 & $710(82.4 \%)$ & $937(81.9 \%)$ & $1,647(82.1 \%)$ \\
E4 & $106(12.3 \%)$ & $128(11.2 \%)$ & $234(11.7 \%)$ \\
E2/E2 & I (0.2\%) & $8(1.4 \%)$ & $8(0.9 \%)$ \\
E2/E3 & $28(6.5 \%)$ & $47(8.2 \%)$ & $75(7.5 \%)$ \\
E2/E4 & $16(3.7 \%)$ & $16(2.8 \%)$ & $32(3.2 \%)$ \\
E3/E3 & $302(70.1 \%)$ & $392(68.6 \%)$ & $695(69.2 \%)$ \\
E3/E4 & $78(18.1 \%)$ & $106(18.5 \%)$ & $184(18.3 \%)$ \\
E4/E4 & $6(1.4 \%)$ & $3(0.5 \%)$ & $9(0.9 \%)$ \\
\hline
\end{tabular}

and that of E2 and E4 was $6.2 \%$ and $11.7 \%$, respectively, which is comparable with other studies performed in Asian populations. The characteristics of subjects with different $A P O E$ genotypes are shown in Table 2. Age, sex, MiniMental State Examination score, height, weight, body mass index, and history of smoking and drinking percentage did not differ among the different $A P O E$ status groups. Serum TC levels were found to be higher in the $A P O E$ E4 group $(P=0.02)$. However, fasting blood glucose and TG levels showed no significant difference among the three groups.

\section{Associations between APOE genotypes and serum levels of glucose, TG, and TC}

Logistic regression analyses were performed to determine the association of $A P O E$ genotypes with serum levels of glucose, TG, and TC. It was found that a higher level of TC was associated with the $\mathrm{E} 4$ allele (adjusted odds ratio 1.689, 95\% confidence interval 1.223-2.334, $P<0.01)$. However, no association was found between $A P O E \mathrm{E} 4$ status and serum levels of glucose (adjusted odds ratio $0.981,95 \%$ confidence interval $0.720-1.336, P=0.903$ ) or TG (adjusted odds ratio $1.042,95 \%$ confidence interval 0.759-1.429, $P=0.800$ ) (Table 3).

\section{Discussion}

In addition to the aberrant accumulation of $\mathrm{A} \beta$ plaques and neurofibrillary tangles, decreased cerebral glucose metabolism is also observed in vulnerable brain areas of AD patients. Abnormal metabolic change is considered as an early event in AD pathology and is supposed to be involved in other AD neuropathological changes. ${ }^{18}$ As a leading significant risk factor for late-onset $\mathrm{AD}, A P O E$ has been documented to be not only linked with $A \beta$ deposition, but also with decreased cerebral glucose metabolism in the brain. ${ }^{7,19,20}$ Recently, one study proposed a hypothesis that the abnormal glucose hypometabolism in the brain of an early-stage $\mathrm{AD}$ patient might result in lower $A P O E$ lipidation status and further lead to decreased clearance and increased deposition of $\mathrm{A} \beta \cdot^{19}$ Another study using 18 F-fluorodeoxyglucose positron emission tomography documented that higher serum glucose levels in cognitively normal, nondiabetic adults were associated with cerebral hypometabolism in vulnerable brain areas of $\mathrm{AD}$ patients. ${ }^{14}$ Furthermore, some researchers proposed that $\mathrm{AD}$ was a special form of diabetes that selectively involves the central nervous system. ${ }^{21}$ It is of particular interest to determine the association between $\mathrm{AD}$ and diabetes from the perspective of AD prevention and therapy. Both $A P O E$ and higher serum glucose levels have been considered to be implicated in $\mathrm{AD}$ and clearly identifying their relationships would help us to better understand the reciprocal effects of $\mathrm{AD}$ and diabetes. However, whether there is any correlation between $A P O E$ genotypes and serum glucose levels remains to be elucidated.

In the current study, a cross-sectional study was performed to determine the correlations between $A P O E$ genotypes and serum levels of glucose, TC, and TG in a cognitively normal, nondiabetic aging population. Great heterogeneity of $A P O E$

Table 2 Characteristics of subjects with different APOE groups

\begin{tabular}{|c|c|c|c|c|}
\hline Characteristics & APOE E2 & APOE E3 & APOE E4 & $P$ \\
\hline N (\%) & $84(8.4 \%)$ & $694(69.2 \%)$ & $225(22.4 \%)$ & NS \\
\hline Age (range), years & $74.57 \pm 0.67(66-92)$ & $73.17 \pm 0.22(64-94)$ & $73.70 \pm 0.40(65-90)$ & NS \\
\hline Sex, male/female & $29 / 55$ & $302 / 392$ & $100 / 125$ & NS \\
\hline Height, cm & $|57.38 \pm 0.9|$ & $157.55 \pm 0.32$ & $158.10 \pm 1.43$ & NS \\
\hline Weight, kg & $57.49 \pm 1.29$ & $58.75 \pm 0.44$ & $57.68 \pm 0.76$ & NS \\
\hline $\mathrm{BMI}, \mathrm{kg} / \mathrm{m}^{2}$ & $23.13 \pm 0.44$ & $23.58 \pm 0.16$ & $23.6 I \pm 0.26$ & NS \\
\hline Fasting blood glucose, $\mathrm{mmol} / \mathrm{L}$ & $5.18 \pm 0.12$ & $5.27 \pm 0.04$ & $5.30 \pm 0.08$ & NS \\
\hline Serum triglycerides, mmol/L & $1.53 \pm 0.15$ & $1.52 \pm 0.03$ & $1.54 \pm 0.07$ & NS \\
\hline Total cholesterol, mmol/L & $5.10 \pm 0.12$ & $5.07 \pm 0.04$ & $5.27 \pm 0.07$ & 0.02 \\
\hline Alcohol users ( $>$ I drink/week) & $12(14.3 \%)$ & $117(16.9 \%)$ & 49 (21.8\%) & NS \\
\hline Cigarette smoking & $12(14.2 \%)$ & 132 (19.0\%) & $50(22.2 \%)$ & NS \\
\hline
\end{tabular}

Note: Three groups were divided according to APOE genotypes: E2/2 or E2/3 (APOE E2); E3/3 (APOE E3); and E2/4, E3/4, or E4/4 (APOE E4). Abbreviations: BMI, body mass index; NS, not significant. 
Table 3 Adjusted effects of APOE E4 allele on biochemical parameters

\begin{tabular}{llll}
\hline & OR & $\mathbf{9 5 \%} \mathbf{~ C l}$ & $\boldsymbol{P}$ \\
\hline Total cholesterol & $\mathrm{I} .689$ & $1.223-2.334$ & 0.001 \\
Total triglyceride & $\mathrm{I} .042$ & $0.759-1.429$ & 0.800 \\
Glucose & 0.981 & $0.720-1.336$ & 0.903 \\
\hline
\end{tabular}

Note: Logistic regression model was adjusted for age, body mass index, alcohol use, and smoking use.

Abbreviations: $\mathrm{OR}$, odds ratio; $\mathrm{Cl}$, confidence interval.

genotypes has been observed in different populations. The APOE allele frequencies in the current study were comparable with similar studies performed in Chinese populations. ${ }^{5,22,23} \mathrm{It}$ was found that serum TC level was higher in the E4 group compared with the other two groups, and the E4 allele was positively associated with higher TC, which coincides with previous studies. ${ }^{5,6}$

With regard to the association between $A P O E$ genotypes and serum levels of glucose, one study has reported that E4 carriers had lower glucose levels compared with E3/ E3 carriers in females..$^{24}$ However, no association was found between the $A P O E \mathrm{E} 4$ allele and serum levels of glucose in either males or females in the current study. Previous studies have shown that $A P O E$ might be involved in decreased cerebral glucose metabolism in the brain. ${ }^{20}$ Considering the great difference in glucose metabolism between the central nervous system and peripheral system, it is not surprising that $A P O E$ had no significant effect on peripheral glucose metabolism.

There are several limitations of the current study that need to be addressed. First, only one indicator for peripheral glucose metabolism was used. It might be more accurate if combined with other indicators such as glucose intolerance and insulin resistance. In addition, there was no data on other values of serum lipid parameters such as high-density lipoprotein cholesterol and low-density lipoprotein cholesterol. Also, the sample size was relatively small in the E4 group; future independent studies with a larger sample size are needed to replicate these results.

\section{Conclusion}

This study suggests the following: (1) serum level of TC was higher in the $A P O E$ E4 carrier group; (2) the $A P O E$ E4 allele was positively associated with a high level of serum TC; and (3) no association was found between the $A P O E$ E4 allele and serum levels of glucose and TG in a cognitively normal, nondiabetic aging population, suggesting that the E4 allele may not play a crucial role in peripheral glucose metabolism.

\section{Acknowledgments}

The authors sincerely thank the participants of this study for their cooperation. This work was supported by a grant from the National Natural Science Foundation of China to Zhi-Ying $\mathrm{Wu}(81125009)$ and a grant from the National Natural Science Foundation of Shanghai to Jia-Jun Yang (13ZR1431100).

\section{Disclosure}

The authors report no conflicts of interest in this work.

\section{References}

1. Tanzi RE. The genetics of Alzheimer disease. Cold Spring Harb Perspect Med. 2012;2(10):a006296.

2. Vance JE. Dysregulation of cholesterol balance in the brain: contribution to neurodegenerative diseases. Dis Model Mech. 2012;5(6):746-755.

3. Zlokovic BV. Cerebrovascular effects of apolipoprotein E: implications for Alzheimer disease. JAMA Neurol. 2013;70(4):440-444.

4. Martins IJ, Hone E, Foster JK, et al. Apolipoprotein E, cholesterol metabolism, diabetes, and the convergence of risk factors for Alzheimer's disease and cardiovascular disease. Mol Psychiatry. 2006;11(8):721-736.

5. Liu HC, Hong CJ, Wang SJ, et al. ApoE genotype in relation to AD and cholesterol: a study of 2,326 Chinese adults. Neurology. 1999;53(5):962-966.

6. Raygani AV, Rahimi Z, Kharazi H, Tavilani H, Pourmotabbed T. Association between apolipoprotein E polymorphism and serum lipid and apolipoprotein levels with Alzheimer's disease. Neurosci Lett. 2006;408(1):68-72.

7. Protas HD, Chen K, Langbaum JB, et al. Posterior cingulate glucose metabolism, hippocampal glucose metabolism, and hippocampal volume in cognitively normal, late-middle-aged persons at 3 levels of genetic risk for Alzheimer disease. JAMA Neurol. 2013;70(3):320-325.

8. Dash SK. Cognitive impairment and diabetes. Recent Pat Endocr Metab Immune Drug Discov. 2013;7(2):155-165.

9. Chen Z, Zhong C. Decoding Alzheimer's disease from perturbed cerebral glucose metabolism: implications for diagnostic and therapeutic strategies. Prog Neurobiol. 2013;108:21-43.

10. Crichton GE, Elias MF, Buckley JD, Murphy KJ, Bryan J, Frisardi V. Metabolic syndrome, cognitive performance, and dementia. J Alzheimers Dis. 2012;30(Suppl 2):S77-S87.

11. Crane PK, Walker R, Hubbard RA, et al. Glucose levels and risk of dementia. N Engl J Med. 2013;369(6):540-548.

12. Yaffe K, Falvey C, Hamilton N, et al. Diabetes, glucose control, and 9-year cognitive decline among older adults without dementia. Arch Neurol. 2012;69(9):1170-1175.

13. Kerti L, Witte AV, Winkler A, Grittner U, Rujescu D, Floel A. Higher glucose levels associated with lower memory and reduced hippocampal microstructure. Neurology. 2013;81(20):1746-1752.

14. Burns CM, Chen K, Kaszniak AW, et al. Higher serum glucose levels are associated with cerebral hypometabolism in Alzheimer regions. Neurology. 2013;80(17):1557-1564.

15. Bertolucci PH, Brucki SM, Campacci SR, Juliano Y. [The Mini-Mental State Examination in a general population: impact of educational status]. Arq Neuropsiquiatr. 1994;52(1):1-7. Portuguese.

16. Almeida OP. [Mini Mental State Examination and the diagnosis of dementia in Brazil]. Arq Neuropsiquiatr. 1998;56(3B):605-612. Portuguese.

17. Donohoe GG, Salomaki A, Lehtimaki T, Pulkki K, Kairisto V. Rapid identification of apolipoprotein E genotypes by multiplex amplification refractory mutation system PCR and capillary gel electrophoresis. Clin Chem. 1999;45(1):143-146. 
18. Sanabria-Diaz G, Martinez-Montes E, Melie-Garcia L; Alzheimer's Disease Neuroimaging Initiative. Glucose metabolism during resting state reveals abnormal brain networks organization in the Alzheimer's disease and mild cognitive impairment. PloS One. 2013;8(7):e68860.

19. Patil SP, Ballard R, Sanchez S, Osborn J, Santangelo D Jr. ApoE: the link between Alzheimer's-related glucose hypometabolism and A $\beta$ deposition? Med Hypotheses. 2012;78(4):494-496.

20. Ossenkoppele R, van der Flier WM, Zwan MD, et al. Differential effect of $A P O E$ genotype on amyloid load and glucose metabolism in $\mathrm{AD}$ dementia. Neurology. 2013;80(4):359-365.

21. de la Monte SM, Wands JR. Alzheimer's disease is type 3 diabetes: evidence reviewed. J Diabetes Sci Technol. 2008;2(6):1101-1113.
22. Lin SK, Kao JT, Tsai SM, et al. Association of apolipoprotein E genotypes with serum lipid profiles in a healthy population of Taiwan. Ann Clin Lab Sci. 2004;34(4):443-448.

23. Katzman R, Zhang MY, Chen PJ, et al. Effects of apolipoprotein E on dementia and aging in the Shanghai Survey of Dementia. Neurology. 1997;49(3):779-785.

24. Larson IA, Ordovas JM, DeLuca C, Barnard JR, Feussner G, Schaefer EJ. Association of apolipoprotein (Apo) E genotype with plasma apo E levels. Atherosclerosis. 2000;148(2):327-335.
Clinical Interventions in Aging

\section{Publish your work in this journal}

Clinical Interventions in Aging is an international, peer-reviewed journal focusing on evidence-based reports on the value or lack thereof of treatments intended to prevent or delay the onset of maladaptive correlates of aging in human beings. This journal is indexed on PubMed Central, MedLine,

\section{Dovepress}

CAS, Scopus and the Elsevier Bibliographic databases. The manuscript management system is completely online and includes a very quick and fair peer-review system, which is all easy to use. Visit http://www.dovepress. $\mathrm{com} /$ testimonials.php to read real quotes from published authors. 